\title{
Formal synthesis of (-) anisomycin via organocatalysis
}

\author{
Pandurang V. Chouthaiwale, Shriram P. Kotkar, and Arumugam Sudalai* \\ Chemical Engineering \& Process Development Division, National Chemical Laboratory, \\ Pashan Road, PUNE, Maharashtra, INDIA-411008 \\ E-mail: a.sudalai@ncl.res.in
}

\begin{abstract}
An efficient formal synthesis of (-) anisomycin (1), a potent antibiotic agent, has been achieved in good yields and excellent diastereoselectivity. The key steps are proline-catalyzed sequential $\alpha$-amination or $\alpha$-aminoxylation of aldehyde 2 followed by tandem Horner-Wadsworth-Emmons olefination.
\end{abstract}

Keywords: $\alpha$-amination, $\alpha$-aminoxylation, anisomycin, olefination, proline

\section{Introduction}

(-) Anisomycin, (1), an antibiotic isolated from the fermentation broth of Streptomyces sp., exhibits a strong and selective activity against pathogenic protozoa and fungi and has clinically been used with success in the treatment of vaginitis due to trichomonas vaginitis and of amoebic dysentery. ${ }^{1}$ Both anisomycin (1) and its deacetyl derivative have been used as fungicides in the eradication of bean mildew and as inhibitors of other pathogenic fungi in plants ${ }^{2}$ and peptide bond formation on eukaryotic ribosomes. ${ }^{3}$ Several methods are available in literature for the asymmetric synthesis of (-) anisomycin (1); however, most of them use chiral starting materials with a overall low yield due to large number of steps involved. ${ }^{4}$

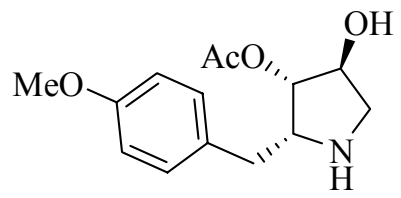

(-)-anisomycin 1

In recent years, organocatalysis has become the main focus of research due to emergence of organocatalyzed tandem transformations ${ }^{5}$ and its application to the synthesis of complex organic molecules that are be accessible in one-pot procedure. As part of our research program aimed at achieving asymmetric synthesis of biologically active molecules using organocatalysts, ${ }^{6}$ herein we report a highly efficient formal synthesis of (-)-anisomycin via L-proline-catalyzed tandem $\alpha$ - 
amination-olefination $^{5 b}$ (Scheme 1) and D-proline-catalyzed sequential $\alpha$-aminoxylationolefination $^{5 \mathrm{a}, 7}$ (Scheme 2) of 3-(4-methyoxyphenyl)propanal (2), a common starting material.

\section{Results and Discussion}

\section{Synthesis of pyrrolidine diol 6 via L-proline-catalyzed sequential $\alpha$-amination-Horner- Wadsworth-Emmons-olefination strategy}

Recently, we have developed a one-pot procedure for the enantioselective synthesis of $\gamma$-amino$\alpha, \beta$-unsaturated esters. ${ }^{5 b}$ We have now made use of this method for achieving the formal synthesis of (-)-anisomycin (1). Accordingly, L-proline-catalyzed sequential $\alpha$-aminationHorner-Wadsworth-Emmons olefination of 3-(4-methoxyphenyl)- propanal (2) was carried out to obtain $\gamma$-amino- $\alpha, \beta$-unsaturated ester 3 in $88 \%$ yield and $99 \%$ ee. The Os-catalyzed diastereoselective dihydroxylation of ester 3 furnished the diol 4 in $85 \%$ yield. Reductive cyclization of 4 was achieved with Raney/Ni $\left(\mathrm{H}_{2}, 60\right.$ psig) in $60 \%$ yield with inseparable mixture of diastereomers ( $\mathrm{dr}$ 7:1 syn:anti as determined by ${ }^{1} \mathrm{H}$ NMR analysis).
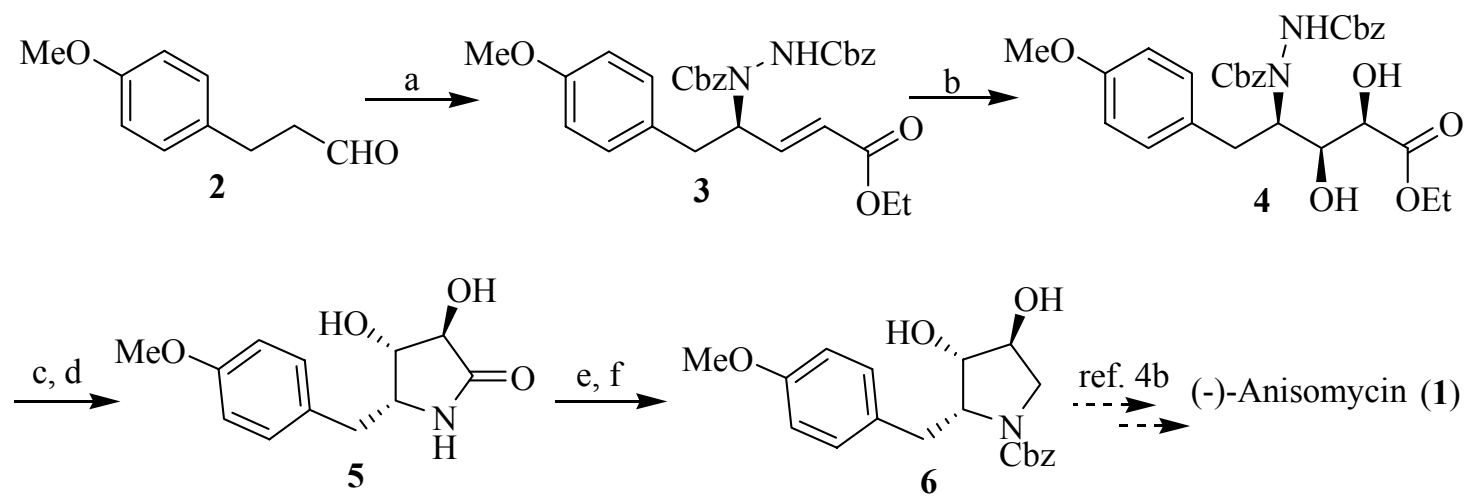

Scheme 1. Reagents and conditions: (a) DBAD, L-proline, $\mathrm{CH}_{3} \mathrm{CN}, 0-10{ }^{\circ} \mathrm{C}, 3 \mathrm{~h}$ then triethyl phosphonoacetate, $\mathrm{LiCl}$, DBU, $5{ }^{\circ} \mathrm{C}$, 45 min., 88 \%; (b) $\mathrm{OsO}_{4}, \mathrm{NMO}$, acetone-water; (c) Raney$\mathrm{Ni}, \mathrm{MeOH}, \mathrm{H}_{2}$ (60 psi), 12 h; (d) EtOH, reflux, 4 h, $60 \%$ (over two steps); (e) $\mathrm{BH}_{3} . \mathrm{THF}, \mathrm{THF}$, reflux, $10 \mathrm{~h}$; (f) aq. $\mathrm{Na}_{2} \mathrm{CO}_{3}, \mathrm{Cbz}-\mathrm{Cl}, \mathrm{CH}_{2} \mathrm{Cl}_{2}, 4 \mathrm{~h}, 66 \%$ (over two steps).

The predominant syn selectivity was determined by NOE study, ${ }^{5 b}$ which is also confirmed by matching the sign of optical rotation of $\mathbf{6}$ with literature values. ${ }^{4 \mathrm{~b}}$ The amide carbonyl in $\mathbf{5}$ was then reduced with $\mathrm{BH}_{3}$. THF; followed by its amine protection gave N-Cbz protected diol $\mathbf{6}$ in 66 $\%$ yield. $[\alpha]_{\mathrm{D}}^{25}-7.3$ (c 1, MeOH) $\left\{\right.$ lit. $^{4 b}[\alpha]_{\mathrm{D}}^{25}-8.2$ (c 5.97, MeOH) $\}$ (Scheme 1). Since a moderate diastereoselectivity for 4 was obtained in this approach, a modified $\alpha$-aminoxylation approach was taken up as described below. 


\section{Synthesis of butyrolactone diol 9 via D-proline-catalyzed sequential $\alpha$-aminoxylation- Horner-Wadsworth-Emmons olefination strategy}

In this approach, the synthesis of 1 was started with $\alpha$-aminoxylation of 3-(4methoxyphenyl)propanal (2), which was carried out using nitrosobenzene as the electrophilic component followed by in situ Horner-Wadsworth-Emmons olefination ${ }^{5 \mathrm{a}, 7}$ with DBU as base that furnished anilinoxy olefinic ester 7 in $79 \%$ yield. The deprotection of anilinoxy group to hydroxy group was achieved with $\mathrm{Cu}(\mathrm{OAc})_{2}$ in ethanol. The optical purity of 8 was found to be $99 \%$ ee as determined by its Mosher ester analysis. The Os-catalyzed diastereoselective dihydroxylation of unsaturated ester 8 furnished lactone diol 9 in $82 \%$ yield with high optical purity as a single diastereomer. $[\alpha]^{25}-73\left(c\right.$ 1, MeOH) $\left\{\right.$ lit. $^{4 p}[\alpha]^{25}-72.2$ (c 0.41, MeOH)\} (Scheme 2). The conversion of pyrrolidine diol 6 and butyrolactone diol 9 into (-)-anisomycin (1) has already been reported. ${ }^{4 b, p}$
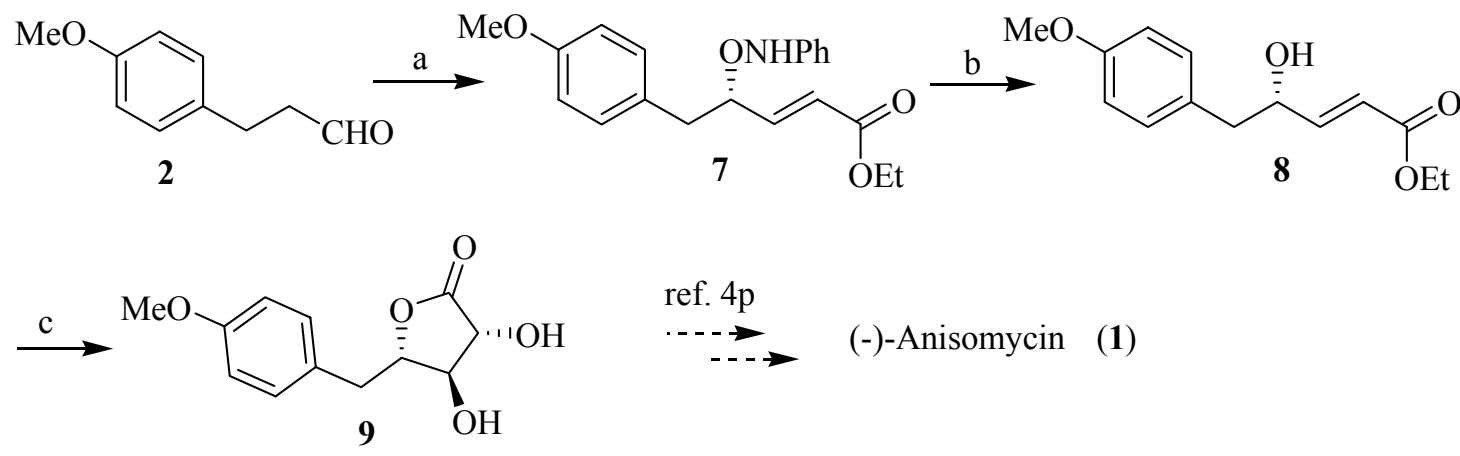

Scheme2. Reagents and conditions (a) PhNO, D-proline (20 mol \%), $\mathrm{CH}_{3} \mathrm{CN},-20{ }^{\circ} \mathrm{C}, 24 \mathrm{~h}$ then triethyl phosphonoacetate, $\mathrm{LiCl}, \mathrm{DBU}, 1 \mathrm{~h}, 79 \%$; (b) $\mathrm{Cu}(\mathrm{OAc})_{2}, \mathrm{EtOH}, 25^{\circ} \mathrm{C}, 12$ h; (c) $\mathrm{OsO}_{4}$, NMO, acetone-water, 2 h, $82 \%$.

\section{Conclusions}

In conclusion, a short, formal synthesis of (-)-anisomycin (1) employing proline-catalyzed sequential $\alpha$-aminooxylation or $\alpha$-amination coupled with tandem Horner-Wadsworth-Emmons olefination of 3-(4-methyoxyphenyl)propanal (2) has been achieved. This is the first organocatalytic route involving operationally simple procedures with high yields and excellent enantioselectivities. The use of inexpensive proline in catalytic amounts and less number of steps render our approach a good alternative to the known methods.

\section{Experimental Section}

General Procedures. Solvents were purified and dried by standard procedures before use. Melting points are uncorrected. Optical rotations were measured using sodium $\mathrm{D}$ line on a JASCO-P-1020 digital polarimeter. ${ }^{1} \mathrm{H}$ and ${ }^{13} \mathrm{C}$ NMR spectra were recorded on Brucker AC-200 
spectrometer. Enantiomeric excess was measured using either the chiral HPLC, Mosher ester analysis or by comparison with specific rotation. Elemental analysis was carried out on a Carlo Erba CHNS-O analyzer.

(2R,3S,4R)-Ethyl 4-(dibenzyloxycarbonylhydrazinyl)-2,3-dihydroxy-5-(4 methoxyphenyl) pentanoate (4). To a solution of olefin $3(1.06 \mathrm{~g}, 2 \mathrm{mmol})$ and 4-Methylmorpholine N-oxide (NMO) (0.702 g, $6 \mathrm{mmol}, 3$ equiv.) in $20 \mathrm{~mL}$ THF- $\mathrm{H}_{2} \mathrm{O}(1: 1)$ at $0{ }^{\circ} \mathrm{C}$, was added $\mathrm{OsO}_{4}(25.4 \mathrm{mg}$, $0.1 \mathrm{M}$ in toluene, $5 \mathrm{~mol} \%$ ) and the reaction mixture was stirred at the same temperature for $12 \mathrm{~h}$ and at $25^{\circ} \mathrm{C}$ for $6 \mathrm{~h}$. The reaction was quenched with sodium bisulfite $(0.5 \mathrm{~g})$, diluted with water and extracted with ethyl acetate $(3 \times 50 \mathrm{~mL})$. The combined organic layers were washed with brine, dried over anhydrous $\mathrm{Na}_{2} \mathrm{SO}_{4}$ and concentrated under reduced pressure to give crude product, which was then purified by flash column chromatography using petroleum ether: ethyl acetate (35:65) to afford pure diol 4.

Yield: $0.962 \mathrm{~g}, 85 \%$; $[\alpha]_{\mathrm{D}}^{25}+21.66\left(c 1.0, \mathrm{CHCl}_{3}\right)$; IR $\left(\mathrm{CHCl}_{3}\right)$ v 3400, 3220, 3020, 2929, 2400, 1658, 1429, 1220, 1075, 923, $758 \mathrm{~cm}^{-1}$; ${ }^{1} \mathrm{H}$ NMR $\left(200 \mathrm{MHz}, \mathrm{CDCl}_{3}+\mathrm{DMSO}_{6}\right) \delta 1.32(\mathrm{t}, J=$ 7.1 Hz, 3H), 2.90-2.99 (m, 1H), 3.14-3.24 (m, 1H), $3.73(\mathrm{~m}, 3 \mathrm{H}), 4.30(\mathrm{~m}, 3 \mathrm{H}), 5.0(\mathrm{~m}, 6 \mathrm{H}), 6.09$ $(\mathrm{m}, 1 \mathrm{H}), 6.63(\mathrm{~d}, J=7.1 \mathrm{~Hz}, 1 \mathrm{H}), 6.74(\mathrm{~d}, J=8.2 \mathrm{~Hz}, 1 \mathrm{H}), 6.97(\mathrm{~d}, J=8.2 \mathrm{~Hz}, 1 \mathrm{H}), 7.07(\mathrm{~d}, J=$ $8.0 \mathrm{~Hz}, 1 \mathrm{H}), 7.28-7.38(\mathrm{~m}, 10 \mathrm{H}) ;{ }^{13} \mathrm{C}$ NMR $\left(50 \mathrm{MHz}, \mathrm{CDCl}_{3}+\mathrm{DMSO}_{-}\right)$) $\delta 13.2,31.8,54.0$, $60.1,66.3,66.5,70.4,70.5,112.5,126.2,126.4,126.7,126.9,127,127.1,127.2,127.3,127.5$, 129.1, 129.3, 134.6, 135, 135.2, 154.7, 155.6, 156.9, 171.7; Elemental analysis: $\mathrm{C}_{30} \mathrm{H}_{34} \mathrm{~N}_{2} \mathrm{O}_{9}$ requires $\mathrm{C}, 63.59 ; \mathrm{H}, 6.05 ; \mathrm{N}, 4.94$; found $\mathrm{C}, 63.35 ; \mathrm{H}, 6.15 ; \mathrm{N}, 4.68 \%$.

(3R,4S,5R)-5-(4-Methoxybenzyl)-3,4-dihydroxypyrrolidin-2-one (5). A solution of diol 4 $(0.849 \mathrm{mg}, 1.5 \mathrm{mmol})$ in $\mathrm{MeOH}(20 \mathrm{~mL})$ and acetic acid $(0.3 \mathrm{ml})$ was treated with Raney nickel ( $3 \mathrm{~g}$, excess) under $\mathrm{H}_{2}$ ( $80 \mathrm{psig}$ ) for $24 \mathrm{~h}$. The reaction mixture was filtered over celite and concentrated to give the crude amino diol, which on stirring in $\mathrm{EtOH}$ at $50{ }^{\circ} \mathrm{C}$ for $4 \mathrm{~h}$ gave the cyclized product 5 (purified by flash chromatography using ethyl acetate as eluent). Yield: 0.213 g, $60 \%$; $[\alpha]^{25}+16.25\left(c 1.0, \mathrm{CHCl}_{3}\right) ; \mathrm{IR}\left(\mathrm{CHCl}_{3}\right) \vee 3330,2920,2864,1670,1463,1377,1225$, 1121, $728 \mathrm{~cm}^{-1}$; ${ }^{1} \mathrm{H}$ NMR $\left(200 \mathrm{MHz}, \mathrm{CDCl}_{3}+\mathrm{DMSO}_{6}\right) \delta 2.52(\mathrm{~m}, 1 \mathrm{H}), 2.94-3.05(\mathrm{~m}, 1 \mathrm{H})$, 3.51 (brs, 2H), 3.68 (m, 1H), 3.75 (s, 3H), 3.82 (d, $J=7.5 \mathrm{~Hz}, 1 \mathrm{H}), 4.11$ (t, $J=7.4 \mathrm{~Hz}, 1 \mathrm{H}), 6.79$ (m, 2H), $7.13(\mathrm{~m}, 2 \mathrm{H}), 7.44(\mathrm{~s}, 1 \mathrm{H}) ;{ }^{13} \mathrm{C}$ NMR $\left(50 \mathrm{MHz}, \mathrm{CDCl}_{3}+\mathrm{DMSO}_{\mathrm{d}}\right): \delta 33.7,53.4$, 54.6, $72.1,73.3,112.0,128.9,129.1,129.2,156.2,172.85$; Elemental analysis: $\mathrm{C}_{12} \mathrm{H}_{15} \mathrm{NO}_{4}$ requires $\mathrm{C}$, $60.75 ; \mathrm{H}, 6.37$; N, 5.90; found C, 60.45; H, 6.15; N, $5.68 \%$.

(2R,3S,4S)-benzyl-2-(4-methoxybenzyl)-3,4-dihydroxypyrrolidine-1-carboxylate (6). To a solution of amide $5(111 \mathrm{mg}, 0.5 \mathrm{mmol})$ in dry THF $(10 \mathrm{~mL})$ was added $\mathrm{BH}_{3}$.DMS $(0.3 \mathrm{~mL}$, excess, $95 \%$ ) and the reaction mixture was refluxed for $10 \mathrm{~h}$. After completion of reaction (TLC) it was quenched with dil. $\mathrm{HCl}$ and solvents were removed under reduced pressure. The crude residue was dissolved in $\mathrm{MeOH}$ and treated with $\mathrm{Et}_{3} \mathrm{~N}(2 \mathrm{~mL})$. The solvents were removed under reduced pressure and the crude product was directly used for the next step without purification. 
To a solution of the above crude amine in dry THF $(10 \mathrm{~mL})$ was added $\mathrm{Na}_{2} \mathrm{CO}_{3}(80 \mathrm{mg}, 0.7$ $\mathrm{mmol})$ at $0{ }^{\circ} \mathrm{C}$, after stirring for $10 \mathrm{~min}$. $\mathrm{Cbz}-\mathrm{Cl}(85 \mathrm{mg}, 0.5 \mathrm{mmol})$ was added and the reaction mixture was stirred at $25{ }^{\circ} \mathrm{C}$ for $2 \mathrm{~h}$. The reaction mixture was extracted with $\mathrm{CHCl}_{3}$, dried over anhydrous $\mathrm{Na}_{2} \mathrm{SO}_{4}$. Evaporation of solvents under reduced pressure followed by column chromatographic purification gave 6 as a colorless solid. Yield: 0.110 g, $66 \%$, mp $126{ }^{\circ} \mathrm{C}$ (lit. ${ }^{4 b}$ mp 127-129 $\left.{ }^{\circ} \mathrm{C}\right) ;[\alpha]^{25}$ D $-7.2\left(c\right.$ 1.0, MeOH) $\left[\right.$ lit. $^{4 b}[\alpha]^{25}$ D -8.2 (c 1.0, MeOH)] ; IR $\left(\mathrm{CHCl}_{3}\right) v$ 3320, 3050, 3022, 2920, 2864, 1670, 1423, 1357, 1235, 1121, $815 \mathrm{~cm}^{-1} ;{ }^{1} \mathrm{H}$ NMR (200 MHz, $\left.\mathrm{CDCl}_{3}\right) \delta 1.75-1.82(\mathrm{~m}, 2 \mathrm{H}), 2.87(\mathrm{~m}, 1 \mathrm{H}), 2.93(\mathrm{~m}, 2 \mathrm{H}), 3.68(\mathrm{~m}, 1 \mathrm{H}), 3.77(\mathrm{~s}, 3 \mathrm{H}), 4.16-4.23$ $(\mathrm{m}, 3 \mathrm{H}), 5.16(\mathrm{~s}, 2 \mathrm{H}), 6.76(\mathrm{~d}, J=8.2 \mathrm{~Hz}, 2 \mathrm{H}), 6.97(\mathrm{~d}, J=8.4 \mathrm{~Hz}, 2 \mathrm{H}), 7.37(\mathrm{~m}, 5 \mathrm{H}) ;{ }^{13} \mathrm{C}$ NMR $\left(50 \mathrm{MHz}, \mathrm{CDCl}_{3}\right): \delta 32.3,40.3,55.1,60.9,68.9,70.0,79.1,113.8,128.1,128.5,129.8,136.2$, 156.7; Elemental analysis: $\mathrm{C}_{20} \mathrm{H}_{23} \mathrm{NO}_{5}$ requires $\mathrm{C}, 67.21 ; \mathrm{H}, 6.49 ; \mathrm{N}, 3.92$; found $\mathrm{C}, 67.55$; $\mathrm{H}$, $6.25 ; \mathrm{N}, 4.73 \%$.

(S)-Ethyl 4-anilinoxy-5-(4-methoxyphenyl)pent-2-enoate (7). To a solution of nitrosobenzene (1 g, $9.3 \mathrm{mmol})$ and D-proline (158 mg, $15 \mathrm{~mol} \%$ ) in $\mathrm{CH}_{3} \mathrm{CN}(20 \mathrm{~mL})$ was added 3-(4methoxyphenyl)propanal $(1.8 \mathrm{~g}, 11.2 \mathrm{mmol})$ at $-20{ }^{\circ} \mathrm{C}$. The reaction mixture was stirred at the same temperature for $24 \mathrm{~h}$ followed by addition of $\mathrm{LiCl}$ (566 mg, 1.5 equiv.), triethyl phosphonoacetate (3.13 g, 1.5 equiv.) and after stirring for $5 \mathrm{~min} \mathrm{DBU}$ (1.4 g, 1 equiv.) was added. The reaction mixture was quenched with half saturated $\mathrm{NH}_{4} \mathrm{Cl}$ and extracted with ethyl acetate $(3 \times 20 \mathrm{~mL})$. Combined organic phases were concentrated and dried over anhydrous $\mathrm{Na}_{2} \mathrm{SO}_{4}$. Purification by flash column chromatography (Pet ether: EtOAc $=85: 15$ ) afforded aminoxy olefinic ester 7. Yield: $3 \mathrm{~g}, 79 \%$; $[\alpha]^{25}-25.0\left(c 1, \mathrm{CHCl}_{3}\right)$; IR $\left(\mathrm{CHCl}_{3}\right) v_{\max } 3016$, 2935, 2839, 2360, 1716, 1600, 1494, 1512, 1247, 1035, $757 \mathrm{~cm}^{-1} ;{ }^{1} \mathrm{H}$ NMR (200 MHz, $\left.\mathrm{CDCl}_{3}\right)$ : $\delta 1.29(\mathrm{t}, J=7.1 \mathrm{~Hz}, 3 \mathrm{H}), 2.81-2.91(\mathrm{dd}, J=6.1,13.9 \mathrm{~Hz}, 1 \mathrm{H}), 2.99-3.09(\mathrm{dd}, J=7.5,14.0 \mathrm{~Hz}$, $1 \mathrm{H}), 3.81(\mathrm{~s}, 3 \mathrm{H}), 4.15$ (q, $J=7.1 \mathrm{~Hz}, 2 \mathrm{H}), 4.53(\mathrm{~m}, 1 \mathrm{H}), 5.95-6.04(\mathrm{dd}, J=1.1,15.8 \mathrm{~Hz}, 1 \mathrm{H})$, $6.68(\mathrm{~d}, J=7.5 \mathrm{~Hz}, 2 \mathrm{H}), 6.84-6.98(\mathrm{~m}, 5 \mathrm{H}), 7.13-7.21(\mathrm{~m}, 4 \mathrm{H}) ;{ }^{13} \mathrm{C} \mathrm{NMR}\left(50 \mathrm{MHz}, \mathrm{CDCl}_{3}\right): \delta$ 14.1, 39.1, 55.2, 60.5, 84.1, 113.8, 114.2, 122.0, 122.9, 128.8, 130.6, 131.9, 146.4, 148.1, 158.3, 166; Elemental analysis: $\mathrm{C}_{20} \mathrm{H}_{23} \mathrm{NO}_{4}$ requires $\mathrm{C}, 70.36 ; \mathrm{H}, 6.79 ; \mathrm{N}, 4.10$; found $\mathrm{C}, 70.63 ; \mathrm{H}$, $6.73 ; \mathrm{N}, 4.57 \%$.

(S)-Ethyl 4-hydroxy-5-(4-methoxyphenyl)pent-2-enoate (8). To a solution of ester 7 (2.5 g, $7.3 \mathrm{mmol})$ in ethanol $(25 \mathrm{~mL})$ was added $\mathrm{Cu}(\mathrm{OAc})_{2}(488 \mathrm{mg}, 0.3$ equiv.) and the reaction mixture was stirred at $25{ }^{\circ} \mathrm{C}$ for $12 \mathrm{~h}$. The reaction mixture was quenched with saturated ammonium chloride solution and extracted with ethyl acetate $(3 \times 50 \mathrm{~mL})$. The combined organic layers were dried over anhydrous $\mathrm{Na}_{2} \mathrm{SO}_{4}$ and condensed under vaccum. The crude product was purified by flash column chromatography (Pet ether: EtOAc $=75: 25)$ to give pure 8 in $70 \%$ yield. Yield: $1.2 \mathrm{~g}, 70 \%$; $[\alpha]^{25}-10.27\left(c 1, \mathrm{CHCl}_{3}\right)$; IR $\left(\mathrm{CHCl}_{3}\right) v_{\max } 3020,2360,2343$, 1716, 1650, 610, 1512, 1247, 1217, 1178, 1037, $757 \mathrm{~cm}^{-1} ;{ }^{1} \mathrm{H}$ NMR $\left(200 \mathrm{MHz}, \mathrm{CDCl}_{3}\right): \delta 1.29$ (t, $J=7.1 \mathrm{~Hz}, 3 \mathrm{H}), 2.34$ (brs, $1 \mathrm{H}), 2.67-2.78(\mathrm{dd}, J=8.1,13.7 \mathrm{~Hz}, 1 \mathrm{H}), 2.84-2.94(\mathrm{dd}, J=5.0$, $13.8 \mathrm{~Hz}, 1 \mathrm{H}), 3.79(\mathrm{~s}, 3 \mathrm{H}), 4.18(\mathrm{q}, J=7.2,2 \mathrm{H}), 4.47(\mathrm{~m}, 1 \mathrm{H}), 6.0(\mathrm{dd}, J=1.6,15.6 \mathrm{~Hz}, 1 \mathrm{H})$, $6.84(\mathrm{~d}, J=8.6,2 \mathrm{H}), 6.94-7.05(\mathrm{dd}, J=4.6,15.6 \mathrm{~Hz}, 1 \mathrm{H}), 7.11(\mathrm{~d}, J=8.6,2 \mathrm{H}) ;{ }^{13} \mathrm{C} \mathrm{NMR}(50$ 
$\left.\mathrm{MHz}, \mathrm{CDCl}_{3}\right): \delta 14.1,42.2,55.1,60.3,71.7,114.0,120.4,128.6,130.4,149.0,158.4,166.4$; Elemental analysis: $\mathrm{C}_{14} \mathrm{H}_{18} \mathrm{O}_{4}$ requires $\mathrm{C}, 67.18 ; \mathrm{H}, 7.25$ found $\mathrm{C}, 67.54 ; \mathrm{H}, 7.62 \%$.

$\mathbf{( 3 R , 4 R , 5 S ) - 5 - ( 4 - m e t h o x y b e n z y l ) - d i h y d r o - 3 , 4 - d i h y d r o x y f u r a n - 2 ( 3 H ) - o n e ~ ( 9 ) . ~ T o ~ a ~ s o l u t i o n ~ o f ~}$ $50 \%$ aq. $N$-methylmorpholine $N$-oxide $(0.93 \mathrm{~mL}, 4 \mathrm{mmol})$ and osmium tetraoxide (101 $\mathrm{mg}, 10$ $\mathrm{mol} \%)$ in acetone $(10 \mathrm{~mL})$ was added a solution of ester $8(1 \mathrm{~g}, 4 \mathrm{mmol})$ in acetone $(10 \mathrm{~mL})$ at 0 ${ }^{\circ} \mathrm{C}$ and the reaction mixture was stirred for $2 \mathrm{~h}$ at the same temperature. To the reaction mixture was added $10 \% \mathrm{Na}_{2} \mathrm{SO}_{3}$ at $0{ }^{\circ} \mathrm{C}$ and was stirred for $30 \mathrm{~min}$. The generated precipitate was filtered through celite pad and the filtrate was concentrated. The residue was diluted with ether and treated with $10 \%$ aq. $\mathrm{HCl}$. The organic layer was dried over anhydrous $\mathrm{Na}_{2} \mathrm{SO}_{4}$ and evaporated to give the crude product 9, which was purified by flash column chromatography (Pet ether: ethyl acetate $=55: 45)$ and recrystallized from $\mathrm{CHCl}_{3}$ Yield: $780 \mathrm{mg}, 82 \%$ yield, colorless solid $\left(\mathrm{mp}=81{ }^{\circ} \mathrm{C}\right.$, lit. $\left.{ }^{4 \mathrm{p}} \mathrm{mp}=81-82{ }^{\circ} \mathrm{C}\right) ;[\alpha]^{25} \mathrm{D}-72.5(c 1, \mathrm{MeOH}),\left[\right.$ lit. $^{4 \mathrm{p}}[\alpha]^{25} \mathrm{D}-72.2(c$ 0.41, $\mathrm{MeOH}) ; \mathrm{IR}\left(\mathrm{CHCl}_{3}\right) v_{\max } 3330,2920,2864,1760,1465,1387,1225,1131,728 \mathrm{~cm}^{-1}$; ${ }^{1} \mathrm{H}$ NMR $\left(200 \mathrm{MHz}, \mathrm{CDCl}_{3}\right): \delta 2.81(\mathrm{dd}, J=7.5,14.7 \mathrm{~Hz}, 1 \mathrm{H}), 3.12(\mathrm{dd}, J=3.3,14.7 \mathrm{~Hz}, 1 \mathrm{H}), 3.79$ (s, $3 \mathrm{H}), 3.98$ (t, $J=8.4,1 \mathrm{H}), 4.22(\mathrm{dt}, J=3.3,7.6 \mathrm{~Hz}, 1 \mathrm{H}$ ), 4.33 (d, $J=8.7 \mathrm{~Hz}, 1 \mathrm{H}$ ), 5.35 (brs, $2 \mathrm{H}), 6.81(\mathrm{~d}, J=8.7 \mathrm{~Hz}, 2 \mathrm{H}), 7.17(\mathrm{~d}, J=8.7,2 \mathrm{H}) ;{ }^{13} \mathrm{C} \mathrm{NMR}\left(50 \mathrm{MHz}, \mathrm{CDCl}_{3}\right): \delta 35.8,54.1$, 73.2 , 75.4, 80.0, 112.7, 127.4, 129.5, 157.2, 173.5; Elemental analysis: $\mathrm{C}_{12} \mathrm{H}_{14} \mathrm{O}_{5}$ requires $\mathrm{C}$, $60.50 ; \mathrm{H}, 5.92$ found $\mathrm{C}, 60.84 ; \mathrm{H}, 5.62 \%$.

\section{Acknowledgements}

PVC and S.P.K. thank CSIR, New Delhi for the award of research fellowships. The authors are thankful to Dr. B. D. Kulkarni, Head, CEPD, for his support and encouragement.

\section{References}

1. (a) Jimenez, A.; Vazquez, D. In Antibiotics; Hahn, F. E., Ed.; Springer: Berlin, 1979; pp 119. (b) Grollmann, A. P. J. Biol. Chem. 1967, 242, 3226. (c) Frye, W. W.; Mule, J. G.; Swartzwelder, C. Antibiot. Ann. 1955, 820.

2. The Merck Index, 12th Ed.; Windholtz, M., Ed.; Merck: Whitehouse Station, NJ, 1996; p 710.

3. Korzybski, T.; Kowsyk-Gindifer, Z.; Kurytowicz, W. In Antibiotics; American Society of Microbiology: Washington, DC, 1978; Vol. 1, pp 343-346.

4. (a) Wong, C. M.; Buccini, J.; Chang, I.; Te Raa, J.; Schwenk, R. Can. J. Chem. 1969, 47, 2421. (b) Iida, H.; Yamazaki, N.; Kibayashi, C. J. Org. Chem. 1986, 51, 1069. (c) Shono, T.; Kise, N. Chem. Lett. 1987, 697. (d) Ballini, R.; Marcantoni, E.; Petrini, M. J. Org. Chem. 1992, 57, 1316. (e) Yoda, H.; Nakajima, T.; Yamazaki, H.; Takabe, K. Heterocycles 1995, 41, 2423. (f) Han, G.; LaPorte, M. G.; McIntosh, M. C.; Weinreb, S. M.; Parvez, M. J. Org. 
Chem. 1996, 61, 9483. (g) Kang, S. H.; Choi, H. -W. J. Chem. Soc., Chem. Commun. 1996, 1521. (h) Kadota, I.; Saya, S.; Yamamoto, Y. Heterocycles 1997, 46, 335. (i) Ono, M.;

Suzuki, K.; Akitha, H. Tetrahedron Lett. 1999, 40, 8223. (j) Delair, P.; Brot, E.; Kanazawa, A.; Greene, A. E. J. Org. Chem. 1999, 64, 1383. (k) Hutin, P.; Haddad, M.; Larcheveque, M. Tetrahedron: Asymmetry 2000, 11, 2547. (1) Chandrasekhar, S.; Ramachander, T.; Reddy, M. V. Synthesis 2002, 1867. (m) Hulme, A. N.; Rosser, E. M. Org. Lett. 2002, 4, 265. (n) Huang, P. -Q.; Zheng, X. ARKIVOC 2003, 7. (o) Chang, M. -Y.; Chen, S. -T.; Chang, N. C. Heterocycles 2003, 60, 1203. (p) Ono, M.; Tanikawa, S.; Suzuki K.; Akita, H.

Tetrahedron 2004, 60, 10187. (q) Hirner, S.; Somfai, P. Synlett 2005, 3099. (r) Reddy, J. S.; Kumar, A. R.; Rao, B. V. Tetrahedron: Asymmetry 2005, 16, 3154.

5. (a) Zhong, G.; Yu, Y. Org. Lett. 2004, 6, 1637. (b) Kotkar, S.P.; Chavan, V. B.; Sudalai, A. Org. Lett. 2007, 9, 1001. (c) Zhao, G. -L.; Liao, W, -W.; Cordova, A. Tetrahedron Lett 2006, 47, 4929.

6. (a) Kotkar, S. P.; Sudalai, A. Tetrahedron Lett. 2006, 47, 6813. (b) Narina, S. V.; Sudalai, A. Tetrahedron Lett. 2006, 47, 6799. (c) Kotkar, S. P.; Sudalai, A. Tetrahedron: Asymmetry 2006, 17, 1738.

7. Mangion, I. K.; MacMillan, D. W. C. J. Am. Chem. Soc. 2005, 127, 3696. 